PROCEEDINGS OF THE

AMERICAN MATHEMATICAL SOCIETY

Volume 125, Number 7, July 1997, Pages 1893-1901

S $0002-9939(97) 04122-1$

\title{
HEREDITARY NOETHERIAN CATEGORIES WITH A TILTING COMPLEX
}

\author{
HELMUT LENZING
}

(Communicated by Eric M. Friedlander)

In memory of Maurice Auslander

\begin{abstract}
We are characterizing the categories of coherent sheaves on a weighted projective line as the small hereditary noetherian categories without projectives and admitting a tilting complex. The paper is related to recent work with de la Peña (Math. Z., to appear) characterizing finite dimensional algebras with a sincere separating tubular family, and further gives a partial answer to a question of Happel, Reiten, Smalø (Mem. Amer. Math. Soc. 120 (1996), no. 575) regarding the characterization of hereditary categories with a tilting object.
\end{abstract}

\section{A CHARACTERIZATION OF WEIGHTED PROJECTIVE LINES}

We are going to characterize the categories $\operatorname{coh}(\mathbb{X})$ of coherent sheaves on a weighted projective line $\mathbb{X}[3,4]$.

Theorem 1. Let $k$ be an algebraically closed field. For a small connected abelian $k$-category $\mathcal{H}$ with finite dimensional morphism and extension spaces the following assertions are equivalent:

(i) $\mathcal{H}$ is equivalent to the category of coherent sheaves on a weighted projective line.

(ii) Each object of $\mathcal{H}$ is noetherian. $\mathcal{H}$ is hereditary, has no non-zero projectives, and admits a tilting complex.

(iii) Each object of $\mathcal{H}$ is noetherian, moreover

(a) There exists an equivalence $\tau: \mathcal{H} \rightarrow \mathcal{H}$ (Auslander-Reiten translation) such that Serre duality $\operatorname{DExt}^{1}(A, B) \cong \operatorname{Hom}(B, \tau A)$ holds functorially in $A, B \in \mathcal{H}$.

(b) The Grothendieck group $\mathrm{K}_{0}(\mathcal{H})$ is finitely generated free, and the Euler form $\langle-,-\rangle: \mathrm{K}_{0}(\mathcal{H}) \times \mathrm{K}_{0}(\mathcal{H}) \rightarrow \mathbb{Z}$ given on classes of objects of $\mathcal{H}$ by $\langle[X],[Y]\rangle=\operatorname{dim}_{k} \operatorname{Hom}(X, Y)-\operatorname{dim}_{k} \operatorname{Ext}^{1}(X, Y)$ is non-degenerate of determinant \pm 1 .

(c) $\mathcal{H}$ has an object without self-extensions which is not of finite length.

Received by the editors February 9, 1995.

1991 Mathematics Subject Classification. Primary 14G14, 16G20; Secondary 18F20, 18E30.

Key words and phrases. Weighted projective line, coherent sheaf, almost-split sequence, Auslander-Reiten theory, finite dimensional algebra, quiver, derived category, exceptional object, tilting complex. 
The result includes a characterization of the category $\operatorname{coh}\left(\mathbb{P}_{1}(k)\right)$ of coherent sheaves on the projective line adding the requirement: If $F$ is not of finite length and $S$ is simple in $\mathcal{H}$, then $\operatorname{Hom}(F, S) \neq 0$. The theorem also gives a partial answer to a question raised by Happel, Reiten, Smalø [6] about the hereditary abelian $k$ categories admitting a tilting object.

For a finite dimensional $k$-algebra $\Sigma, \bmod (\Sigma)$ denotes the category of finite dimensional right modules. For a $k$-vectorspace $X$ we denote its $k$-dual $\operatorname{Hom}_{k}(X, k)$ by $\mathrm{D} X$. For an abelian category $\mathcal{H}$ its derived category of bounded complexes is denoted $D^{b}(\mathcal{H})$, and $X \mapsto X[n]$ denotes the $n$th iterate of the translation functor for $D^{b}(\mathcal{H})$. An object $X$ in $\mathcal{H}$ is called noetherian if each ascending chain of subobjects of $X$ is stationary. We say that $E \in \mathcal{H}$ is without self-extensions if $\operatorname{Ext}^{n}(E, E)=0$ holds for each $n \neq 0$. A $k$-category $\mathcal{H}$ is connected if a representation of $\mathcal{H}$ as a coproduct $\mathcal{H} \cong \mathcal{A} \amalg \mathcal{B}$ implies $\mathcal{A}=0$ or $\mathcal{B}=0$. We say that $\mathcal{H}$ is hereditary if $\operatorname{Ext}_{\mathcal{H}}^{2}(-,-)=0$.

A tilting object $\Sigma$ in $\mathcal{H}$ is defined by the fact that the right derived functor of $\operatorname{Hom}(\Sigma,-): \mathcal{H} \rightarrow \bmod (\operatorname{End}(\Sigma))$ induces an equivalence of triangulated categories $D^{b}(\mathcal{H}) \rightarrow D^{b}(\bmod (\operatorname{End}(\Sigma)))$. More generally, we say that $\mathcal{H}$ admits a tilting complex if for some finite dimensional algebra $\Sigma$ there exists an equivalence $\Phi: D^{b}(\bmod (\Sigma)) \rightarrow D^{b}(\mathcal{H})$ of triangulated categories. Here, $\Sigma$ has an interpretation as the endomorphism ring of the tilting complex $\Phi(\Sigma)$ of $D^{b}(\mathcal{H})$. Whenever convenient, we prefer to view a tilting complex as a full subcategory of $D^{b}(\mathcal{H})$ with finitely many objects, thus avoiding the use of endomorphism rings.

This work has substantially profited from the collaboration with José Antonio de la Peña on finite dimensional algebras with a sincere separating tubular family [9]; we further thank Hagen Meltzer for critical comments on a first draft of this paper.

\section{Proof OF THE CHARACTERIZATION}

Let $\mathcal{H}_{0}$ be the full subcategory consisting of all objects of $\mathcal{H}$ having finite length.

Lemma 1. Assume that $\mathcal{H}$ satisfies condition (iii)(a). Then $\mathcal{H}_{0}$ is uniserial, i.e. each object $U \in \mathcal{H}_{0}$ has a unique finite composition series. Accordingly, for some index set $\mathbb{X}$ the category $\mathcal{H}_{0}$ decomposes into a coproduct $\mathcal{H}_{0}=\coprod_{x \in \mathbb{X}} \mathcal{U}_{x}$ of connected uniserial categories $\mathcal{U}_{x}$, whose indecomposables form stable tubes.

If moreover $\mathrm{K}_{0}(\mathcal{H})$ is finitely generated, then for each $x \in \mathbb{X}$ the simple objects in $\mathcal{U}_{x}$ form an Auslander-Reiten orbit of finite cardinality $u(x)$. Moreover $u(x)=1$ for all but finitely many $x \in \mathbb{X}$.

Proof. In view of (iii)(a) the category $\mathcal{H}$ is hereditary. Hence $\mathcal{H}_{0}$ is a hereditary abelian category with almost-split sequences where each object has finite length, and the Auslander-Reiten translation $\tau: \mathcal{H}_{0} \rightarrow \mathcal{H}_{0}$ is an equivalence. For simple objects $S_{i}, S_{j}$ this implies that $\operatorname{Ext}^{1}\left(S_{j}, S_{i}\right) \neq 0$ if and only if $S_{i} \cong \tau S_{j}$, moreover that in this case the Ext-space is one-dimensional. Uniseriality of $\mathcal{H}_{0}$ now follows easily [2].

For each $x \in \mathbb{X}$ we fix a simple object $S_{x}$ from $\mathcal{U}_{x}$, and collect a representative system $\left(S_{\alpha}\right), \alpha \in I$, of the remaining simple objects. $I$ admits a linear ordering such that $\left\langle\left[S_{\alpha}\right],\left[S_{\beta}\right]\right\rangle=1$ for $\alpha=\beta$, but is zero for $\alpha>\beta$. This implies that the classes $\left[S_{\alpha}\right], \alpha \in I$, are linearly independent in $\mathrm{K}_{0}(\mathcal{H})$, and proves the claim on $u$. 
Proof of (ii) $\Rightarrow$ (iii). In view of $D^{b}(\mathcal{H}) \cong D^{b}(\bmod (\Sigma))$ the group $\mathrm{K}_{0}(\mathcal{H})$ is isomorphic to $\mathrm{K}_{0}(\bmod (\Sigma))$, which is finitely generated free. As a tilting complex for a hereditary category, the algebra $\Sigma$ has finite global dimension which implies the assertion on the Euler form. Happel's theorem [5] shows that $D^{b}(\mathcal{H})=D^{b}(\bmod (\Sigma))$ has Auslander-Reiten triangles, and that further the Auslander-Reiten translation $\tau$ is an equivalence for $D^{b}(\mathcal{H})$. Next we prove that $\tau(\mathcal{H})$ is contained in $\mathcal{H}$. Let $X$ be indecomposable in $\mathcal{H}$, we get $\tau X=Y[n]$ for some $Y \in \mathcal{H}$ and $n \in \mathbb{Z}$. Because

$$
\operatorname{DExt}_{\mathcal{H}}^{n+1}(X, Y) \cong \operatorname{Hom}_{D^{b}(\mathcal{H})}(Y[n+1], \tau X[1]) \neq 0,
$$

$n \geq 1$ is impossible for a hereditary category. Assuming $n \leq-1$ implies

$$
\operatorname{DExt}_{\mathcal{H}}^{1}(X, Z) \cong \operatorname{Hom}_{D^{b}(\mathcal{H})}(Z, Y[n])=0
$$

for each $Z \in \mathcal{H}$, so leads to a non-zero projective $X$ in $\mathcal{H}$. This proves the assertion on Serre duality for $\mathcal{H}$, which in turn implies the existence of almost-split sequences in $\mathcal{H}$ (cf. [3]).

Finally assume that condition (iii) (c) is violated. Then $D^{b}(\mathcal{H})$ has a tilting complex lying in $D^{b}\left(\mathcal{H}_{0}\right)$, which involving Lemma 1 implies that $\mathcal{H}=\mathcal{H}_{0}$ is connected uniserial, hence the existence of a simple object $S$ of $\tau$-period $p$ such that the classes $[S],[\tau S], \ldots,\left[\tau^{p-1} S\right]$ form a $\mathbb{Z}$-basis of $\mathrm{K}_{0}(\mathcal{H})$. Then $\mathbf{a}=\sum_{j=0}^{p-1}\left[\tau^{j} S\right]$ is a non-zero element of $\mathrm{K}_{0}(\mathcal{H})$ with $\langle\mathbf{a},-\rangle=0$, contradiction.

The proof of implication (iii) $\Rightarrow$ (i) will occupy the rest of the paper. Each object $X$ from $\mathcal{H}$ has a largest subobject $t X$ of finite length (its torsion part), and $X / t X$ has no simple submodules, i.e. is torsionfree. The full subcategory of torsionfree objects is denoted $\mathcal{H}_{+}$.

Lemma 2. An indecomposable object $X$ of $\mathcal{H}$ is either torsion $\left(X \in \mathcal{H}_{0}\right)$ or torsionfree $\left(X \in \mathcal{H}_{+}\right)$.

Proof. Since $\operatorname{DExt}^{1}(X / t X, t X)=\operatorname{Hom}\left(\tau^{-}(t X), X / t X\right)=0$ the sequence $0 \rightarrow$ $t X \rightarrow X \rightarrow X / t X \rightarrow 0$ splits.

Lemma 3. There is a surjective linear mapping $\mathrm{rk}: \mathrm{K}_{0}(\mathcal{H}) \rightarrow \mathbb{Z},[X] \mapsto \operatorname{rk} X$, called rank function, such that $\operatorname{rk} \tau X=\operatorname{rk} X$ for each $X \in \mathcal{H}$, rk vanishes on $\mathcal{H}_{0}$, and $\operatorname{rk} F>0$ for each $0 \neq F \in \mathcal{H}_{+}$.

Proof (de la Peña). For $x \in \mathbb{X}$ and $S_{x} \in \mathcal{U}_{x}$ simple, we define $w_{x}=\sum_{j=1}^{\mathcal{U}(x)}\left[\tau^{j} S_{x}\right]$ in $\mathrm{K}_{0}(\mathcal{H})$. Note that each $w_{x}$ is stable under the mapping induced by $\tau$ on $\mathrm{K}_{0}(\mathcal{H})$. We choose $x_{1}, \ldots, x_{s}$ from $\mathbb{X}$ such that the subgroup of $\mathrm{K}_{0}(\mathcal{H})$ generated by $w_{x_{1}}, \ldots, w_{x_{s}}$ contains all $w_{x}(x \in \mathbb{X})$ and put $w=w_{x_{1}}+\cdots+w_{x_{s}}$.

By noetherianness each non-zero $F \in \mathcal{H}_{+}$has a simple quotient $S_{x}$, hence $\left\langle[F], w_{x}\right\rangle>0$, which implies $\left\langle[F], w_{x_{i}}\right\rangle>0$ for some $i=1, \ldots, s$, and finally $\langle[F], w\rangle>0$. If $\varepsilon$ denotes the index of $\left\langle\mathrm{K}_{0}(\mathcal{H}), w\right\rangle$ in $\mathbb{Z}$ we may take rk $\mathbf{a}=$ $1 / \varepsilon \cdot\langle\mathbf{a}, w\rangle$.

Lemma 4. Assume $B \in \mathcal{H}_{+}$and $\operatorname{Ext}^{1}\left(\mathcal{U}_{x}, B\right) \neq 0$. For each $n \geq 0$ there exists an exact sequence $0 \rightarrow B \rightarrow B_{n} \rightarrow U_{n} \rightarrow 0$ with $B_{n} \in \mathcal{H}_{+}, U_{n} \in \mathcal{U}_{x}$ and $\left[U_{n}\right]=n \cdot w_{x}$.

Proof. Let $S_{x}$ be simple in $\mathcal{U}_{x}$ with $\operatorname{Ext}^{1}\left(S_{x}, B\right) \neq 0$. Choose non-split exact sequences $0 \rightarrow B_{i}^{\prime} \rightarrow B_{i+1}^{\prime} \rightarrow \tau^{-i} S_{x} \rightarrow 0(i=0,1,2, \ldots)$, where $B_{0}^{\prime}=B$, and put $B_{n}=B_{n u(x)}^{\prime}$. 
Lemma 5. For each non-zero $F$ in $\mathcal{H}_{+}$and each $x \in \mathbb{X}$ there is a non-zero homomorphism from $F$ into an object from $\mathcal{U}_{x}$. Accordingly, $[X]=0$ in $\mathrm{K}_{0}(\mathcal{H})$ implies $X=0$ in $\mathcal{H}$.

Proof. We put

$$
\begin{aligned}
& \mathbb{Y}=\left\{y \in \mathbb{X} \mid \operatorname{Hom}\left(F, \mathcal{U}_{y}\right)=0\right\}, \\
& \mathcal{A}=\left\{A \in \mathcal{H} \mid \operatorname{Hom}\left(A, \mathcal{U}_{y}\right)=0 \text { for all } y \in \mathbb{Y}\right\}, \\
& \mathcal{B}=\{B \in \mathcal{H} \mid \operatorname{Hom}(\mathcal{A}, B)=0\} .
\end{aligned}
$$

Step $1 . \operatorname{Hom}\left(\mathcal{B}, \mathcal{U}_{x}\right)=0$ for all $x \in \mathbb{X} \backslash \mathbb{Y}$.

Since $\tau \mathcal{U}_{x}=\mathcal{U}_{x}$ it is equivalent to prove that $\operatorname{Ext}^{1}\left(\mathcal{U}_{x}, \mathcal{B}\right)=0$. Otherwise we obtain some $B$ in $\mathcal{B}$ and exact sequences

$$
0 \rightarrow B \rightarrow B_{n} \rightarrow U_{n} \rightarrow 0 \quad(n=1,2, \ldots),
$$

having the properties of Lemma 4 . We show that each $B_{n}$ actually belongs to $\mathcal{B}$. Consider a morphism $f: A \rightarrow B_{n}$ with $A \in \mathcal{A}$, and from the pull-back

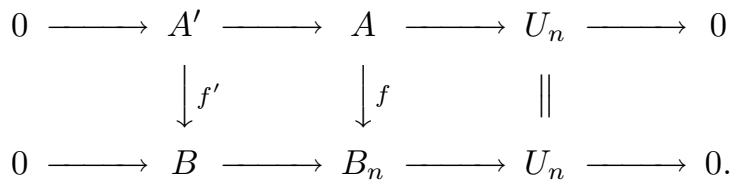

Using $x \notin \mathbb{Y}$ we see that $A^{\prime} \in \mathcal{A}$, hence $f^{\prime}=0$, so $f$ induces a morphism $U_{n} \rightarrow B_{n}$ which is zero, implying $\operatorname{Hom}\left(\mathcal{A}, B_{n}\right)=0$. Since $\left[B_{n}\right]=[B]+n w_{x}$, we obtain $\left\langle[F], B_{n}\right\rangle>0$ for large $n$, thus contradicting $\operatorname{Hom}(\mathcal{A}, \mathcal{B})=0$.

Step 2. Each $X$ in $\mathcal{H}$ has the form $X \cong A \oplus B$ with $A \in \mathcal{A}, B \in \mathcal{B}$.

By noetherianness $X$ has a maximal subobject $A$ from $\mathcal{A}$, accordingly $X / A$ has no non-zero subobject from $\mathcal{A}$, hence belongs to $\mathcal{B}$. Obviously, $\mathcal{A}$ is closed under $\tau^{-}$, therefore $\operatorname{DExt}^{1}(X / A, A)=\operatorname{Hom}\left(\tau^{-} A, X / A\right)=0$, and $X \cong A \oplus X / A$.

Step 3. $\operatorname{Hom}(\mathcal{B}, \mathcal{A})=0$.

Otherwise we find $A \in \mathcal{A} \cap \mathcal{H}_{+}, B \in \mathcal{B} \cap \mathcal{H}_{+}$and a non-zero morphism $f: B \rightarrow A$. Let $A^{\prime}=\operatorname{im}(f)$. We choose $A^{\prime} \subseteq A^{\prime \prime} \subseteq A$ such that $A^{\prime \prime} / A^{\prime}=t\left(A / A^{\prime}\right)$. Since $A \in \mathcal{A}$ and $A / A^{\prime \prime} \in \mathcal{H}_{+}$we see that $A^{\prime \prime} \in \mathcal{A}$. Replacing $A$ by $A^{\prime \prime}$ we may thus assume that $A / A^{\prime} \in \mathcal{H}_{0}$, in fact $A / A^{\prime} \in \coprod_{x \in \mathbb{X} \backslash \mathbb{Y}} \mathcal{U}_{x}$. By Step 1 the sequence $0 \rightarrow A^{\prime} \rightarrow A \rightarrow A / A^{\prime} \rightarrow 0$ splits, so $A^{\prime}$ belongs to $\mathcal{A}$. We thus arrive at an epimorphism $B \rightarrow A^{\prime} \rightarrow S_{x}$ where $S_{x}$ is simple in $\mathcal{U}_{x}$ and $x \in \mathbb{X} \backslash \mathbb{Y}$, contradiction, which proves the assertion of Step 3 .

Combining Steps 2 and 3 with $\operatorname{Hom}(\mathcal{A}, \mathcal{B})=0$ we see that $\mathcal{H}=\mathcal{A} \amalg \mathcal{B}$. Since $\mathcal{H}$ is connected and $0 \neq F \in \mathcal{A}$, it follows that $\mathcal{B}=0$, hence $\mathbb{Y}=\varnothing$ as claimed.

By $\mathrm{K}_{0}^{\prime}(\mathcal{H})$ we denote the image of the natural map $\mathrm{K}_{0}\left(\mathcal{H}_{0}\right) \rightarrow \mathrm{K}_{0}(\mathcal{H})$, i.e. the subgroup of $\mathrm{K}_{0}(\mathcal{H})$ generated by the classes of simple objects.

Lemma 6. There exists a torsionfree object $L$ of rank one. $\mathrm{K}_{0}(\mathcal{H})=\mathbb{Z}[L] \oplus \mathrm{K}_{0}^{\prime}(\mathcal{H})$. Moreover for any $x, y \in \mathbb{X}, w_{x}$ and $w_{y}$ are proportional.

Proof. Choose a torsionfree object $L \neq 0$ of minimal rank. Each $\mathbf{a} \in \mathrm{K}_{0}^{\prime}(\mathcal{H})$ satisfies $\operatorname{rk} \mathbf{a}=0$, on the other hand $\operatorname{rk} L>0$, which implies $\mathbb{Z}[L] \cap \mathrm{K}_{0}^{\prime}(\mathcal{H})=0$. We show by induction on $r=\operatorname{rk} A$ that the class $[A]$ of an indecomposable object $A$ belongs 
to $\mathbb{Z}[L]+\mathrm{K}_{0}^{\prime}(\mathcal{H})$. For $r=0$ this follows from Lemma 3. For $r>0, A$ is torsionfree. We choose $x \in \mathbb{X}$ and a simple object $S_{x}$ from $\mathcal{U}_{x}$ with $\operatorname{Ext}^{1}\left(S_{x}, A\right) \neq 0$. Invoking Lemma 4 we get an exact sequence $0 \rightarrow A \rightarrow \bar{A} \rightarrow U \rightarrow 0$, where $\bar{A}$ is torsionfree, $U$ is in $\mathcal{U}_{x}$, and a non-zero homomorphism $f: L \rightarrow \bar{A}$. Because $L$ has minimal rank and $\bar{A}$ is torsionfree, $f$ is a monomorphism hence $C=\operatorname{coker}(f)$ has strictly smaller rank than $A$. By induction $[C]$, hence $[A]$, belongs to $\mathbb{Z}[L] \oplus \mathrm{K}_{0}^{\prime}(\mathcal{H})$, proving the splitting of $\mathrm{K}_{0}(\mathcal{H})$. Surjectivity of the rank function now implies that $L$ has rank one.

Since the linear forms $\left\langle-, w_{x}\right\rangle$ and $\left\langle-, w_{y}\right\rangle$ vanish on $\mathrm{K}_{0}^{\prime}(\mathcal{H})$ they are determined by their non-zero value on $[L]$, hence are proportional. Because the Euler form on $\mathrm{K}_{0}(\mathcal{H})$ is non-degenerate, the corresponding assertion holds for $w_{x}$ and $w_{y}$.

Through the formation of perpendicular categories [4] we are going to reduce the weight function $u: \mathbb{X} \rightarrow \mathbb{N}$. An object $E$ from $\mathcal{H}$ is called exceptional if $E$ has a trivial endomorphism ring $k$ and no self-extensions. Invoking Lemma 5 it follows from [7] that each indecomposable object $E \in \mathcal{H}$ without self-extensions is exceptional. Notice that every simple object $S$ in $\mathcal{U}_{x}$ with $u(x)>1$ is exceptional.

Proposition 1. Let $x \in \mathbb{X}$ be exceptional, i.e. $u(x)>1$, and let $S$ be simple in $\mathcal{U}_{x}$.

(a) The right perpendicular category $\mathcal{H}^{\prime}=S^{\perp}$ of $S$ in $\mathcal{H}$, consisting of all $X \in \mathcal{H}$ with $\operatorname{Hom}(S, X)=0=\operatorname{Ext}^{1}(S, X)$, satisfies conditions (iii) (a), (b), (c) again.

(b) $\mathbb{X}$ serves naturally also as a parametrizing set for the decomposition of $\mathcal{H}_{0}^{\prime}=S^{\perp} \cap \mathcal{H}_{0}$ into connected uniserial categories $\mathcal{U}_{y}^{\prime}=S^{\perp} \cap \mathcal{U}_{y}$. The number of isomorphism classes of simples from $\mathcal{U}_{y}^{\prime}$ is given by $u^{\prime}(x)=u(x)-1$ and $u^{\prime}(y)=u(y)$ for $y \neq x$. Moreover, inclusion $\mathcal{H}^{\prime} \hookrightarrow \mathcal{H}$ preserves the rank.

(c) $\mathrm{K}_{0}(\mathcal{H})=\mathbb{Z}[S] \oplus \mathrm{K}_{0}\left(\mathcal{H}^{\prime}\right)$.

Proof. Since $\mathcal{H}$ is hereditary and $S$ is exceptional, inclusion $\mathcal{H}^{\prime} \hookrightarrow \mathcal{H}$ admits a left adjoint $\ell$ and a right adjoint $r$ [4]. Since $S$ is simple, the category $\mathcal{H}_{0}^{\prime}$ of torsion objects in $\mathcal{H}^{\prime}$ agrees with $\mathcal{H}^{\prime} \cap \mathcal{H}_{0}$, and therefore $\mathcal{H}_{0}^{\prime}=\coprod_{y \in \mathbb{X}} \mathcal{U}_{y}^{\prime}$ with $\mathcal{U}_{y}^{\prime}=\mathcal{U}_{y}$ if $y \neq x$; further $\mathcal{U}_{x}^{\prime}=S^{\perp} \cap \mathcal{U}_{x}$ is connected as well with $u(x)-1$ isomorphic classes of simple objects. Moreover, $w_{y}^{\prime}=w_{y}$ for each $y \in \mathbb{X}$, so inclusion $\mathcal{H}^{\prime} \hookrightarrow \mathcal{H}$ preserves the rank.

If $E$ is torsionfree in $\mathcal{H}$ without self-extensions, then $\operatorname{Hom}(S, E)=0$ and $\ell E$ is given by the middle term of the $S$-universal extension

$$
0 \rightarrow E \rightarrow \ell E \rightarrow \operatorname{Ext}^{1}(S, E) \otimes_{k} S \rightarrow 0
$$

in particular $\ell E$ is non-zero torsionfree. From the exactness of

$$
0=\operatorname{Ext}^{1}(E, E) \rightarrow \operatorname{Ext}^{1}(E, \ell E) \rightarrow \operatorname{Ext}^{1}(S, E) \otimes_{k} \operatorname{Ext}^{1}(E, S)=0
$$

we get $\operatorname{Ext}^{1}(E, \ell E)=0$. By definition $\operatorname{Ext}^{1}(S, \ell E)=0$ holds, hence $\operatorname{Ext}^{1}(\ell E, \ell E)=$ 0 , which proves the existence of a torsionfree object in $\mathcal{H}^{\prime}$ without self-extensions.

Finally, assume that $A$ and $B$ are in $\mathcal{H}^{\prime}$. Since

$$
\operatorname{DExt}^{1}(A, B)=\operatorname{Hom}\left(\ell \tau_{\mathcal{H}}^{-} B, A\right)=\operatorname{Hom}\left(B, r \tau_{\mathcal{H}} A\right),
$$

projectivity of $A$ (resp. injectivity of $B$ ) in $\mathcal{H}^{\prime}$ implies $r \tau_{\mathcal{H}} A=0$ (resp. $\ell \tau_{\mathcal{H}}^{-} B=0$ ) which, by the construction of $r$ and $\ell$ [4], implies $A \in \mathcal{H}_{0}^{\prime}$ (resp. $B \in \mathcal{H}_{0}^{\prime}$ ). But any projective (resp. injective) in $\mathcal{U}_{y}^{\prime}, y \in \mathbb{X}$, is zero, hence $A=0$ (resp. $B=0$ ). Invoking Auslander-Reiten theory this implies that $\tau_{\mathcal{H}^{\prime}}=r \tau_{\mathcal{H}}$ and $\tau_{\mathcal{H}^{\prime}}^{-}=\ell \tau_{\mathcal{H}}^{-}$are inverse equivalences. For assertion (c) we refer to [4]. Further $\left\langle[S], \mathrm{K}_{0}\left(\mathcal{H}^{\prime}\right)\right\rangle=0$ implies that the Euler forms on $\mathrm{K}_{0}(\mathcal{H})$ and $\mathrm{K}_{0}\left(\mathcal{H}^{\prime}\right)$ do have the same determinant. 
Repeating the process of Proposition $1 \sum_{x \in \mathbb{X}}(u(x)-1)$ times leads to an exact abelian subcategory $\mathcal{H}^{\prime}$ of $\mathcal{H}$ satisfying (iii) (a), (b), (c) with $u^{\prime}(x)=1$ for each $x \in \mathbb{X}$.

Proposition 2. Each torsionfree rank one object $L$ of $\mathcal{H}^{\prime}$ is exceptional. For each simple object $S_{x}, x \in \mathbb{X}$, we have $\operatorname{dim}_{k} \operatorname{Hom}\left(L, S_{x}\right)=1$ and $\left[S_{x}\right]=w$.

Moreover there exist torsionfree rank one objects $L_{1}, L_{2}$ such that

$$
\operatorname{Hom}\left(L_{2}, L_{1}\right)=0, \quad \operatorname{dim}_{k} \operatorname{Hom}\left(L_{1}, L_{2}\right)=2, \quad \operatorname{Ext}^{1}\left(L_{i}, L_{j}\right)=0 \quad \text { for } i, j=1,2 .
$$

Proof. By Lemma 6 the group $\mathrm{K}_{0}\left(\mathcal{H}^{\prime}\right)$ is free abelian of rank two. Fix a torsionfree object $L$ of rank one, note that $\operatorname{End}(L)=k$ hence $\langle[L],[L]\rangle=1-g$, where $g=$ $\operatorname{dim}_{k} \operatorname{Ext}^{1}(L, L)$, and define the degree function $\operatorname{deg}: \mathrm{K}_{0}\left(\mathcal{H}^{\prime}\right) \rightarrow \mathbb{Z}$ by

$$
\operatorname{deg} z=\langle[L], z\rangle-(1-g) \operatorname{rk} z .
$$

We thus obtain a Riemann-Roch formula

$$
\langle\mathbf{a}, \mathbf{b}\rangle=(1-g) \operatorname{rk} \mathbf{a r k} \mathbf{b}+\left|\begin{array}{cc}
\operatorname{rk} \mathbf{a} & \operatorname{rk} \mathbf{b} \\
\operatorname{deg} \mathbf{a} & \operatorname{deg} \mathbf{b}
\end{array}\right| \quad \text { for all } \mathbf{a}, \mathbf{b} \in \mathrm{K}_{0}\left(\mathcal{H}^{\prime}\right) .
$$

The existence of an exceptional object $E$ of $\mathcal{H}^{\prime}$ implies $0<\langle[E],[E]\rangle=(1-g)(\mathrm{rk} E)^{2}$ hence $g=0$, and so $L$ is exceptional.

Next we choose $x \in \mathbb{X}$ such that $d=\operatorname{deg} w_{x}^{\prime}$ is minimal. Note that $d>0$. For $y \in \mathbb{X}$ write $\operatorname{deg} w_{y}^{\prime}=d \cdot q+r$ with $q, r \in \mathbb{N}$ and $0 \leq r<d$. Extending $L$ by $S_{y}$ and $q$ times by $S_{x}$ we obtain torsionfree rank one objects $L^{\prime}, L^{\prime \prime}$ with

$$
\left[L^{\prime}\right]=[L]+w_{y}^{\prime}, \quad\left[L^{\prime \prime}\right]=[L]+q \cdot w_{x}^{\prime},
$$

hence

$$
\left\langle\left[L^{\prime \prime}\right],\left[L^{\prime}\right]\right\rangle=\langle[L],[L]\rangle+\left\langle[L], w_{y}^{\prime}\right\rangle-q \cdot\left\langle[L], w_{x}\right\rangle \geq 1 .
$$

We thus arrive at an exact sequence $0 \rightarrow L^{\prime \prime} \rightarrow L^{\prime} \rightarrow U \rightarrow 0$, where $U \in \mathcal{H}_{0}^{\prime}$ has degree $<d$. This shows $U=0$, hence $w_{y}^{\prime}=q \cdot w_{x}^{\prime}$. In view of Lemma 6 the classes $[L],\left[S_{x}\right]$ thus form a basis of $\mathrm{K}_{0}\left(\mathcal{H}^{\prime}\right)$, accordingly the determinant of the Euler form is $\pm 1=\left\langle[L],\left[S_{x}\right]\right\rangle^{2}$, proving $\operatorname{dim} \operatorname{Hom}\left(L, S_{x}\right)=1$, therefore $\operatorname{deg} S_{x}=1$.

Put $L_{1}=L$, and let $L_{2}$ be the middle term of a non-split exact sequence $\mu: 0 \rightarrow$ $L_{1} \stackrel{\xi}{\rightarrow} L_{2} \rightarrow S_{x} \rightarrow 0$. Application of $\operatorname{Hom}\left(L_{1},-\right)$ to $\mu$ shows $\operatorname{Hom}\left(L_{1}, L_{2}\right) \cong k^{2}$ and $\operatorname{Ext}^{1}\left(L_{1}, L_{2}\right)=0$, while application of $\operatorname{Hom}\left(-, L_{1}\right)$ leads to $\operatorname{Hom}\left(L_{2}, L_{1}\right)=0$ and $\operatorname{Ext}^{1}\left(L_{2}, L_{1}\right)=0$.

Extend $\xi$ to a $k$-basis $\xi, \eta$ of $\operatorname{Hom}\left(L_{1}, L_{2}\right)$. Invoking the properties of rank and degree, for each $a \in k$ the cokernel $T_{a}$ of $\eta-a \xi: L_{1} \rightarrow L_{2}$ is seen to be simple. In fact, each simple object $S_{y}(y \neq x)$ is isomorphic to some $T_{a}$ : Setting $\xi^{*}=\operatorname{Hom}\left(\xi, S_{y}\right), \eta^{*}=\operatorname{Hom}\left(\eta, S_{y}\right)$ we see that $\xi^{*}$ is an isomorphism because $x \neq y$; moreover, since $k$ is algebraically closed there exists an eigenvalue $a \in k$ such that the kernel $\operatorname{Hom}\left(T_{a}, S_{y}\right)$ of $\eta^{*}-a \xi^{*}: \operatorname{Hom}\left(L_{2}, S_{y}\right) \rightarrow \operatorname{Hom}\left(L_{1}, S_{y}\right)$ is non-zero. Hence $S_{y} \cong T_{a}$, in particular $\left[S_{y}\right]=\left[L_{1}\right]-\left[L_{2}\right]=\left[S_{x}\right]$. 
Proposition 3. $\mathcal{H}$ is a tilting object $\Delta$

$$
\begin{aligned}
& S_{1}^{\left[p_{1}-1\right]} \quad-\cdots \quad-S_{1}^{[1]} \\
& \eta_{1} \\
& L_{1} \frac{\xi_{1}}{\frac{\xi_{2}}{\xi_{2}}} L_{2} \quad \stackrel{\eta_{2}}{\rightarrow} \quad S_{2}^{\left[p_{2}-1\right]} \quad-\cdots \quad-S_{2}^{[1]} \\
& \eta_{t} \\
& S_{t}^{\left[p_{t}-1\right]} \quad-\cdots \quad-S_{t}^{[1]}
\end{aligned}
$$

where $L_{1}, L_{2}$ are torsionfree rank one objects satisfying (1), and $S_{i}^{[l]}$ is an exceptional torsion object of length $l$.

$\Delta$ is a squid algebra in the sense of [10], i.e. equivalent to the path category of the above quiver subject to the relations

$$
\eta_{1} \circ \xi_{1}=0, \quad \eta_{2} \circ \xi_{2}=0, \quad \eta_{i} \circ\left(\xi_{2}-\lambda_{i} \xi_{1}\right)=0, \quad i=3, \ldots, t,
$$

where $\lambda_{3}, \ldots, \lambda_{t}$ are pairwise distinct non-zero elements from $k$.

$\Delta$ also has a realization as a tilting object on the weighted projective line $\mathbb{X}(\mathbf{p}, \boldsymbol{\lambda})$ given by the data $\mathbf{p}=\left(p_{1}, \ldots, p_{t}\right), \boldsymbol{\lambda}=\left(\lambda_{3}, \ldots, \lambda_{t}\right)$.

Proof. Let $x_{1}, \ldots, x_{t}$ denote the sequence of pairwise distinct exceptional elements from $\mathbb{X}$. We put $p_{i}=u\left(x_{i}\right), \mathcal{U}_{i}=\mathcal{U}_{x_{i}}$. Let $S_{i}$ be simple in $\mathcal{U}_{i}$ and $\mathcal{H}^{\prime}$ denote the right perpendicular category with respect to the system

$$
\tau^{l} S_{i}, \quad l=0, \ldots, p_{i}-2, i=1, \ldots, t,
$$

and take a system $L_{1}, L_{2}$ of torsionfree rank one objects from $\mathcal{H}^{\prime}$ as established in Proposition 2.

By induction on the rank it is easily verified (compare proof of Lemma 6) that each object from $\mathcal{H}$ is contained in the smallest subcategory of $\mathcal{H}$ which is closed under extensions, kernels of epimorphisms, cokernels of monomorphisms and contains

$$
L_{1}, L_{2}, S_{i}, \tau S_{i}, \ldots, \tau^{p_{i}-2} S_{i}, \quad i=1, \ldots, t
$$

in particular (4) generates $D^{b}(\mathcal{H})$.

Let $S_{i}^{[n]}$ denote the indecomposable object of $\mathcal{U}_{i}$ with top $S_{i}$ and length $n$. Then the system

$$
L_{1}, L_{2}, S_{i}=S_{i}^{[1]}, S_{i}^{[2]}, \ldots, S_{i}^{\left[p_{i}-1\right]}, \quad i=1, \ldots, t,
$$

generates $D^{b}(\mathcal{H})$ as well, moreover satisfies

$$
\operatorname{Ext}^{1}\left(S_{i}^{[a]}, S_{i^{\prime}}^{\left[a^{\prime}\right]}\right)=0, \quad \operatorname{Ext}^{1}\left(S_{i}^{[a]}, L_{j}\right)=0, \quad \operatorname{Ext}^{1}\left(L_{j}, S_{i}^{[a]}\right)=0
$$

for all $i, i^{\prime}=1, \ldots, t, a=1, \ldots, p_{i}-1, a^{\prime}=1, \ldots, p_{i^{\prime}}-1, j=1,2$. 
Invoking $w_{x}=w$ for each $x \in \mathbb{X}$, we obtain exact sequences

$$
0 \rightarrow L_{1} \stackrel{\xi_{i}}{\rightarrow} L_{2} \stackrel{\bar{\eta}_{i}}{\rightarrow} S_{i}^{\left[p_{i}\right]} \rightarrow 0 \quad(1 \leq i \leq t)
$$

defining $t$ mutually disjoint 1-dimensional subspaces $k \xi_{i}$ of $\operatorname{Hom}\left(L_{1}, L_{2}\right)$, thus $\xi_{i}=$ $\xi_{2}-\lambda_{i} \xi_{1}$ for $2 \leq i \leq t$, where $0=\lambda_{2}, \lambda_{3}, \ldots, \lambda_{t}$ are pairwise distinct. Setting $\eta_{i}=\left[L_{2} \stackrel{\bar{\eta}_{i}}{\rightarrow} S_{i}^{\left[p_{i}\right]} \rightarrow S_{i}^{\left[p_{i}-1\right]}\right]$ and calculating dimensions of Hom-spaces by means of the Euler form further establishes that (2) is a complete set of relations for the subcategory $\Delta$.

It is known (cf. [8]) and not difficult to prove that $\Delta$ can be realized as a tilting sheaf on the weighted projective line $\mathbb{X}(\mathbf{p}, \boldsymbol{\lambda})$ arising from the above data $\mathbf{p}=$ $\left(p_{1}, \ldots, p_{t}\right)$ and $\boldsymbol{\lambda}=\left(\lambda_{3}, \ldots, \lambda_{t}\right)[3]$. Since moreover $\Delta$ has finite global dimension (actually gl. $\operatorname{dim} \Delta \leq 2$ ) we may invoke Beilinson's lemma [1] to conclude that $\Delta$ is also a tilting object in $\mathcal{H}$.

Proof of (iii) $\Rightarrow$ (i). Abbreviating $\mathbb{X}(\mathbf{p}, \boldsymbol{\lambda})$ to $\mathbb{X}$ from now on, the right derived functor of $\operatorname{Hom}_{\mathbb{X}}(\Delta,-): \operatorname{coh}(\mathbb{X}) \rightarrow \bmod (\Delta)$ induces an equivalence of triangulated categories $\alpha: D^{b}(\operatorname{coh}(\mathbb{X})) \rightarrow D^{b}(\bmod (\Delta))$, similarly the right derived functor of $\operatorname{Hom}_{\mathcal{H}}(\Delta,-)$ induces an equivalence $\beta: D^{b}(\mathcal{H}) \rightarrow D^{b}(\bmod (\Delta))$. Since $\alpha$ and $\beta$ preserve $\Delta$ the composition $\phi=\alpha^{-1} \circ \beta: D^{b}(\mathcal{H}) \rightarrow D^{b}(\operatorname{coh}(\mathbb{X}))$ preserves the rank and maps $\mathcal{H}_{0}$ onto the category $\operatorname{coh}_{0}(\mathbb{X})$ of finite length sheaves.

An indecomposable object $X$ from $D^{b}(\mathcal{H})$ lies in $\mathcal{H}$ if and only if $X$ is either in $\mathcal{H}_{0}$ or has rank $>0$ and satisfies $\operatorname{Hom}\left(X, \mathcal{H}_{0}\right) \neq 0$. Similarly an indecomposable object $Y$ from $D^{b}(\operatorname{coh}(\mathbb{X}))$ belongs to $\operatorname{coh}(\mathbb{X})$ if and only if either $Y$ is in $\operatorname{coh}_{0}(\mathbb{X})$ or $Y$ has rank $>0$ and satisfies $\operatorname{Hom}\left(Y, \operatorname{coh}_{0}(\mathbb{X})\right) \neq 0$. Because $\phi\left(\mathcal{H}_{0}\right)=\operatorname{coh}_{0}(\mathbb{X})$, and $\phi$ preserves the rank, it hence follows that $\phi$ induces an equivalence $\mathcal{H} \rightarrow \operatorname{coh}(\mathbb{X})$, thus concluding the proof of the theorem.

Given $\mathcal{H}$ satisfying (ii) or (iii), the theorem implies the existence of a "natural" bijection between the set $\mathbb{X}$, parametrizing the decomposition of $\mathcal{H}_{0}$ into connected components, and the projective line $\mathbb{P}_{1}(k)$, the point set underlying $\mathbb{X}$. To determine the weighted projective line corresponding to $\mathcal{H}$, one fixes a simple object $S$ with $\tau S \cong S$ further a rank one object $L$ of $\mathcal{H}$, and forms a non-split extension $0 \rightarrow$ $L \rightarrow \bar{L} \rightarrow S \rightarrow 0$. The $k$-space $\operatorname{Hom}(L, \bar{L})$ has dimension two; each non-zero $v: L \rightarrow \bar{L}$ is a monomorphism whose cokernel is indecomposable of finite length, therefore belongs to $\mathcal{U}_{\psi(v)}$ for a unique $\psi(v) \in \mathbb{X}$. The induced mapping $\mathbb{P}_{1}(k)=$ $\mathbb{P}(\operatorname{Hom}(L, \bar{L})) \rightarrow \mathbb{X}, k v \mapsto \psi(v)$, is a bijection turning the function $u$ from Lemma 1 into a weight function on $\mathbb{P}_{1}(k)$, determining thus completely the data for the weighted projective line.

\section{REFERENCES}

1. A. A. Beilinson, Coherent sheaves on $P_{n}$ and problems of linear algebra, Funct. Anal. Appl. 12 (1979), 214-216. MR 80c:14010b

2. P. Gabriel, Indecomposable representations II, Symposia Mat. Inst. Naz. Alta Mat. 11 (1973), 81-104. MR 49:5132

3. W. Geigle and H. Lenzing, A class of weighted projective curves arising in representation theory of finite dimensional algebras. In Singularities, representations of algebras, and vector bundles. Lect. Notes Math. 1273 (1987), 265-297, Springer-Verlag, Berlin-Heidelberg-New York. MR 89b:14049

4. _ Perpendicular categories with applications to representations and sheaves, J. Algebra 144 (1991), 273-343. MR 93b:16011 
5. D. Happel, Triangulated categories in the representation theory of finite dimensional algebras, London Math. Soc. Lect. Notes 119, Cambridge University Press, 1988. MR 89e:16035

6. D. Happel, I. Reiten and S. Smalø, Tilting in abelian categories and quasitilted algebras, Mem. Amer. Math. Soc. 120 (1996), no. 575. CMP 96:08

7. D. Happel and C. M. Ringel, Tilted Algebras, Trans. Amer. Math. Soc. 274 (1982), 399-443. MR 84d: 16027

8. H. Lenzing and H. Meltzer, Tilting sheaves and concealed-canonical algebras, CMS Conf. Proc. 18 (1996), 455-473. CMP 96:12

9. H. Lenzing and J. A. de la Peña, Algebras with a separating tubular family, Math. Z., to appear.

10. C. M. Ringel, The canonical algebras with an appendix by William Crawley-Boevey. In Topics in Algebra, Banach Center Publ. 26 (1990), 407-432. MR 93e:16022

Universität-GH Paderborn, Fachbereich Mathematik-Informatik, D-33095 PaderBORN, GeRMANY

E-mail address: helmut@uni-paderborn.de 